\title{
Evaluation of factors affecting 90-day mortality in patients hospitalized due to pulmonary thromboembolism
}

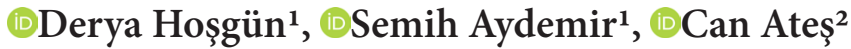 \\ ${ }^{1}$ Atatürk Chest Diseases and Chest Surgery Education and Research Hospital, Department of Intensive Care Unit, Ankara, Turkey \\ ${ }^{2}$ Aksaray University, Faculty of Medicine Department of Biostatistics, Aksaray, Turkey
}

Cite this article as: Hoşgün D, Aydemir S, Ateş C. Evaluation of factors affecting 90-day mortality in patients hospitalized due to pulmonary thromboembolism. J Health Sci Med 2021; 4(5): 543-551.

\begin{abstract}
Aim: Pulmonary thromboembolism (PTE) is a leading cause of death from vascular events. In the pathophysiology of PTE, inflammatory mediators have been shown to be upregulated and to interact with coagulation factors. In this study, we aimed to investigate the role of symptoms, clinical and radiological findings, and the blood parameters measured at presentation within the first $24 \mathrm{~h}$ after the onset of the symptoms in predicting 90 -day mortality and intensive care unit (ICU) requirement in patients with PTE.
\end{abstract}

Material and Method: The retrospective study included 264 PTE patients that were followed up at our Chest Diseases clinic and ICU between 2014 and 2019.

Results: The 264 patients comprised $55.3 \%$ women and $44.7 \%$ men with a mean age of $62.80 \pm 15.95$ years. Of these, 189 of them were hospitalized in the Chest Diseases clinic and the remaining 75 patients were followed up at ICU. Total mortality occurred in $8(3 \%)$ out of 264 patients. Hospital and ICU mortality were determined at $1.9 \%, 1.1 \%$ respectively. The patients comprised 206 (78.0\%) nonmassive PTE, 17 (6.5\%) submassive PTE, and 41 (15.5\%) massive PTE. Risk factors for 90-mortality included white blood cell count (WBC), red blood cell distribution width (RDW), mean platelet volume (MPV)/RDW ratio, right ventricular dilatation (RVD), recombinant tissue plasminogen activator (rtPA) therapy, ICU hospitalization, and increased APACHE II (Acute Physiology and Chronic Health Evaluation II) scores $(\mathrm{p}<0.05)$.

Conclusion: The results indicated that TTE findings, baseline hemodynamic parameters and symptoms, rtPA therapy, and CBC parameters including WBC, NE, and RDW are significant risk factors for predicting both mortality and ICU requirement.

Keywords: Pulmonary embolism, inflammatory, mortality

\section{INTRODUCTION}

In acute PTE, early diagnosis and treatment are of prime importance in the reduction of mortality, and the mortality rates range from 0.3 to 12 per 100 diagnoses (1-3). Right ventricular dilatation (RVD) is partially associated with early mortality and can be evaluated by transthoracic echocardiography (TTE) and spiral thoracic computed tomography pulmonary angiography (CTPA). In the pathophysiology of PTE, inflammatory mediators have been shown to be upregulated and serum levels of inflammatory markers including C-reactive protein (CRP) and interleukin-6 (IL-6) have been shown to be elevated within $48 \mathrm{~h}$ after the onset of acute thrombosis (4).

Red blood cell distribution width (RDW), mean platelet volume (MPV), total neutrophil count (NE), and total lymphocyte count (LY) are inexpensive and standard

biomarkers routinely measured in complete blood count (CBC). The percentage of serum RDW typically increases in hemoglobinopathies associated with iron deficiency. Additionally, recent clinical studies indicated that the percentage of serum RDW also increases in patients with increased oxidative stress and inflammation (5). On the other hand, RDW has been shown to be a useful marker in the diagnosis and prediction of prognosis and mortality in PTE patients. MPV, which is also measured in CBC, represents average platelet size and correlates with platelet function and activation. Although the MPV level in bone marrow may decrease or increase depending on the levels of inflammatory cytokines, it remains unknown as to which conditions cause increased or decreased serum MPV levels (6). Additionally, although serum MPV levels have been shown to be higher in patients with deep vein 
thrombosis (DVT) compared to control subjects, there is no standardization in the literature regarding serum levels of MPV in PTE patients (7). In the presence of inflammation, NE is increased while LY is decreased as a result of elevated cortisol levels caused by the activation of sympathetic system. Moreover, both the neutrophil-tolymphocyte ratio (NLR) and the platelet-to-lymphocyte ratio (PLR) have been shown to be higher in PTE patients compared to non-PTE individuals $(8,9)$.

The present study was designed to investigate the role of symptoms, clinical and TTE findings, and the blood parameters measured at presentation in predicting 90-day mortality and intensive care unit (ICU) requirement in patients that were diagnosed with PTE in our emergency service and then referred to the Chest Diseases clinic or ICU for treatment within the first $24 \mathrm{~h}$ after presentation.

\section{MATERIAL AND METHOD}

The retrospective study included 264 PTE patients that were followed up at our Chest Diseases clinic and intensive care unit (ICU) between 2014 and 2019. After obtaining an ethical approval from the Pamukkale University Noninterventional Clinical Research Ethics Committee (Date: 21.05.2019, Decision No: 10). Health Sciences University, Atatürk Chest Diseases and Chest Surgery Education and Research Hospital, Specialization in Medicine Education Board approval (Date: 27.02.2020, Decision No: 664). All procedures were carried out in accordance with the ethical rules and the principles of the Declaration of Helsinki. Patient records, ICU observation charts, and plain radiographic images were retrieved from hospital databases and were reviewed for each patient. All the patients included in the study initially presented to our emergency service and were transferred to the chest diseases clinic or ICU within the first $24 \mathrm{~h}$ after presentation, underwent $\mathrm{CBC}$ examination at presentation, and were initiated on 90-day mortality follow-up and on appropriate treatments in accordance with international guidelines. Additionally, the findings of both CTPA and 2-point compression ultrasonography of the lower extremity (USG) were evaluated by an experienced radiologist and the diagnosis of PTE was established by an experienced cardiologist based on TTE findings. Due to our retrospective study, USG could not be standardized. At the same time, it could not be repeated in the 3-5-7 days recommended in the guidelines for the diagnosis of DVT (1). The TTE findings were classified in terms of RVD, paradoxical septal motion with left-axis deviation, and pulmonary artery pressure (PAP). Based on hemodynamic monitoring findings, a heart rate of $50-100$ beats/min was accepted as normal, $>100$ beats/min as tachycardia, and $<50$ beats/min as bradycardia. In noninvasive arterial blood pressure, a systolic blood pressure(SBP) of 90-120 mmHg was accepted as normotensive, a $>20 \mathrm{mmHg}$ increase in SBP compared to baseline values or a SBP of $>120 \mathrm{mmHg}$ was accepted as hypertension, and a $\leq 40 \mathrm{mmHg}$ decrease in SBP within 15 min compared to baseline values or a SBP of $<90 \mathrm{mmHg}$ was accepted as hypotension. Oxygen saturation measured by pulse oximetry $\left(\mathrm{SpO}_{2}\right)>90 \%$ was considered as normal. $\mathrm{SpO}_{2}<90 \%$ was accepted as hypoxemia $(1,2)$. Exclusion criteria were as follow: pregnancy, age below 18 years, signs of active infection, chronic kidney disease, malignancies, connective tissue disease, ongoing anticoagulant therapy, myocardial infarction, hematological malignances, history of blood transfusion within the last one month, thyroid disease, low iron, vitamin B12, and folic acid levels, and an ongoing treatment. CTPA was performed using a 128-slice CT device (slice thickness, $0.6 \mathrm{~mm}$ ) (Definition AS, Siemens Medical Solutions, Forchheim, Germany). CBC parameters were measured by the photometric method on a Mindray BC-800 autoanalyzer. Normal ranges of CBC parameters including white blood cell count (WBC), hemoglobin (HB), hematocrit (HCT), platelet count (PLT), RDW, NE, LY, and MPV were accepted as 4.6-10.2 $10^{3} / \mu \mathrm{L}, 12-16 \mathrm{~g} / \mathrm{dL}, 40-54 \%, 142-42410^{3} / \mu \mathrm{L}, 11.6-17.2 \%$, 2-7 $10^{3} / \mu \mathrm{L}, 0.8-410^{3} / \mu \mathrm{L}$, and $7.8-11 \mathrm{fL}$, respectively. Based on CBC measurements, NLR, PLR, and the MPV/PLT and MPV/RDW ratios were calculated for each patient.

\section{Statistical Analysis}

Data were summarized as mean \pm standard deviation and median (Range) for continuous variables, frequencies (percentiles) for categorical variables.Student's test or Mann Whitney U test was used for independent group comparisons, depending on the distributional properties of the data.Chi-square test was used for proportions and its counterpart Fisher's Exact test was used when the data were sparse. All analyses were performed IBM SPSS Statistics for Windows, Version 20.0. A p value $<0.05$ was considered as statistically significant.

\section{RESULTS}

The 264 patients comprised 146 (55.3\%) women and 118 $(44.7 \%)$ men with a mean age of $62.80 \pm 15.95$ years. Of these, 189 (71.6\%) of them were hospitalized in the Chest Diseases clinic and the remaining $75(28.4 \%)$ patients were followed up at ICU. In the patients that were hospitalized in ICU, the mean APACHE II (Acute Physiology and Chronic Health Evaluation II) score was 21.40 \pm 5.42 (Table 1). Table 2 presents the findings of CTPA and 2-point compression ultrasonography of the lower extremity.

On TTE examination, RVD was detected in 124 (47.0\%) and paradoxical septal motion with left-axis deviation was detected in 19 (7.3\%) patients, and the mean PAP was $45.90 \pm 15.01 \mathrm{mmHg}$. Table 3 presents the TTE findings for Chest Disease and ICU clinic. 


\begin{tabular}{|c|c|c|c|c|c|}
\hline \multicolumn{2}{|l|}{ Variables } & Chest Disease Clinic Mean \pm SD & ICU Mean \pm SD & Total Mean \pm SD & $\mathbf{p}$ \\
\hline \multicolumn{2}{|l|}{ Age } & $62.69 \pm 15.85$ & $63.07 \pm 16.31$ & $62.80 \pm 15.95$ & 0.864 \\
\hline \multicolumn{2}{|l|}{ Variables } & Chest Diseases Clinic (n, \%) & ICU (n, \%) & Total (n, \%) & $\mathbf{p}$ \\
\hline \multirow{2}{*}{ Gender } & Female & $105(71.9)$ & $41(28.1)$ & $146(55.3)$ & \multirow{2}{*}{0.896} \\
\hline & Male & $84(71.2)$ & $34(28.8)$ & $118(44.7)$ & \\
\hline \multirow{2}{*}{ Comorbiditie } & No & $93(75.6)$ & $30(24.4)$ & $123(46.6)$ & \multirow{2}{*}{0.176} \\
\hline & Yes & $96(68.1)$ & $45(31.9)$ & $141(53.4)$ & \\
\hline \multirow{2}{*}{$\mathrm{DM}$} & No & $170(72.3)$ & $65(27.7)$ & $235(89.0)$ & \multirow{2}{*}{0.442} \\
\hline & Yes & $19(65.5)$ & $10(34.5)$ & $29(11.0)$ & \\
\hline \multirow{2}{*}{ CAD } & No & $142(73.6)$ & $51(26.4)$ & $193(73.1)$ & \multirow{2}{*}{0.239} \\
\hline & Yes & $47(66.2)$ & $24(33.8)$ & $71(26.9)$ & \\
\hline \multirow{2}{*}{$\mathrm{CHF}$} & No & $172(72.3$ & $66(27.7)$ & $238(90.2)$ & \multirow{2}{*}{0.460} \\
\hline & Yes & $17(65.4)$ & $9(34.6)$ & $26(9.8)$ & \\
\hline \multirow{2}{*}{$\mathrm{AF}$} & No & $181(71.3)$ & $73(28.7)$ & 254 (93.9) & \multirow{2}{*}{0.548} \\
\hline & Yes & $8(80.0)$ & $2(20.0)$ & $10(6.1)$ & \\
\hline \multirow{2}{*}{ COPD } & No & $145(69.4)$ & $64(30.6)$ & $209(79.2)$ & \multirow{2}{*}{0.120} \\
\hline & Yes & $44(23.3)$ & $11(14.7)$ & $55(20.8)$ & \\
\hline \multirow{2}{*}{ Dyspnea } & No & $25(51.0)$ & $24(49.0)$ & 49 (18.6) & \multirow{2}{*}{$<0.001$} \\
\hline & Yes & $164(76.3)$ & $51(23.7)$ & $215(81.4)$ & \\
\hline$\Gamma$ & No & $156(72.2)$ & $60(27.8)$ & $216(81.8)$ & 0620 \\
\hline Fever & Yes & $33(68.8)$ & $15(31.3)$ & $48(18.2)$ & 0.629 \\
\hline Dus colve & No & $156(75.7)$ & $50(24.3)$ & $206(78.0)$ & \\
\hline Dry cough & Yes & $33(68.8)$ & $15(31.3)$ & $48(18.2)$ & 0.005 \\
\hline Hemontuci & No & $158(72.8))$ & $59(27.2)$ & $217(82.2)$ & 0245 \\
\hline Hemoptysis & Yes & $31(66.0)$ & $16(34.0)$ & $47(17.8)$ & 0.345 \\
\hline Chest moin & No & $139(73.2)$ & $51(26.8)$ & $190(82.2)$ & 0366 \\
\hline Chest pain & Yes & $50(67.6)$ & $24(32.4)$ & $74(28.0)$ & 0.366 \\
\hline Risk factor & No & $119(82.6)$ & $25(17.4)$ & $144(54.5)$ & \\
\hline & Yes & $70(58.3)$ & $50(41.7)$ & $120(44.5)$ & $<0.001$ \\
\hline Genetic risk & No & $186(71.3)$ & $75(28.7)$ & $261(98.9)$ & \\
\hline factor & Yes & $3(100.0)$ & $0(0.0)$ & $3(11.0)$ & 0.272 \\
\hline Acquired risk & No & $119(83.2)$ & $24(16.8)$ & $143(54.2)$ & \\
\hline factor & Yes & $70(57.9)$ & $51(42.1)$ & $121(81.4)$ & $<0.001$ \\
\hline Variables & & & n & $\%$ & \\
\hline & & No & 236 & 89.4 & \\
\hline Mechanical ve & tilation & NIV & 13 & 4.92 & \\
\hline & & IMV & 15 & 5.68 & \\
\hline Mr & & No & 256 & 97.0 & \\
\hline Mortality (90 & & Yes & 8 & 3.0 & \\
\hline & & No & 261 & 98.9 & \\
\hline Hospital Mor & & Yes & 3 & 1.1 & \\
\hline ICUT Moralit & & No & 259 & 98.1 & \\
\hline ICU Mortality & & Yes & 5 & 1.9 & \\
\hline & & Nonmassive & 206 & 78.0 & \\
\hline Acute PTE & & Massive & 41 & 15.5 & \\
\hline & & Submassive & 17 & 6.5 & \\
\hline & & Normotensive (SBP 90-120 mmHg) & 216 & 81.8 & \\
\hline $\mathrm{SSBP}(\mathrm{mmHg}$ & & $\begin{array}{l}\text { Hypertensive (SBP }>120 \mathrm{mmHg} \text { or }>20 \\
\text { mmHg increase compared to baseline) }\end{array}$ & 4 & 1.51 & \\
\hline & & $\begin{array}{l}\text { Hypotensive (SBP }<90 \mathrm{mmHg} \text { or a } \leq 40 \\
\text { mmHG decrease in SBP within } 15 \mathrm{~min} \\
\text { compared to baseline) }\end{array}$ & 44 & 16.7 & \\
\hline & & Normal (50-100 beats/min) & 124 & 47.0 & \\
\hline Heart rate (be & $\mathrm{s} / \mathrm{min})$ & Tachycardic (>100 beats/min) & 131 & 49.6 & \\
\hline & & Bradycardic $(<50$ beats/min $)$ & 9 & 3.4 & \\
\hline & & Normal $(>\% 90)$ & 97 & 36.7 & \\
\hline $\mathrm{SpO}_{2}(\%)$ & & Hypoxemic $(<\% 90)$ & 167 & 63.3 & \\
\hline
\end{tabular}

DM:Diabetes mellitus, CAD:Coronary artery disease, CHF:Congestive heart failure, AF:Atrial fibrillation, COPD:Chronic obstructive pulmonary disease SBP:Systolic blood pressure, NIV:Noninvasive mechanical ventilation, IMV:Invasive mechanical ventilation, SpO2: Oxygen saturation measured by pulse oximetry, ICU:Intensive care unit,SD: Standard deviation, PTE: Pulmonary thromboembolism. 


\begin{tabular}{|c|c|c|c|}
\hline \multicolumn{2}{|c|}{ Variables } & \multirow{2}{*}{$\begin{array}{c}\text { Frequency } \\
33\end{array}$} & \multirow{2}{*}{$\begin{array}{c}\text { Percentage } \\
12.5\end{array}$} \\
\hline \multirow{11}{*}{ U్ } & Left DVT & & \\
\hline & Right DVT & 14 & 5.3 \\
\hline & Bilateral DVT & 7 & 2.7 \\
\hline & Right main femoral vein DVT & 10 & 3.8 \\
\hline & Left main femoral vein DVT & 7 & 2.7 \\
\hline & Right iliac+femoral vein DVT & 1 & 0.4 \\
\hline & Right main femoral vein+left DVT & 1 & 0.4 \\
\hline & Bilateral iliocaval DVT & 1 & 0.4 \\
\hline & Left iliac vein DVT & 1 & 0.4 \\
\hline & No DVT & 186 & 70.5 \\
\hline & Total & 264 & 100.0 \\
\hline \multirow{11}{*}{ 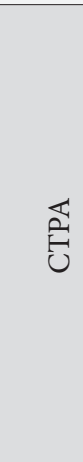 } & Main pulmonary artery thrombus & 14 & 5.3 \\
\hline & Bilateral main pulmonary artery thrombus & 27 & 10.2 \\
\hline & Bilateral segmental artery thrombus & 7 & 2.7 \\
\hline & Bilateral subsegmental artery thrombus & 94 & 35.6 \\
\hline & Right main pulmonary artery thrombus & 20 & 7.6 \\
\hline & Right subsegmental artery thrombus & 67 & 25.4 \\
\hline & Left main pulmonary artery thrombus & 5 & 1.9 \\
\hline & Left subsegmental artery thrombus & 25 & 9.5 \\
\hline & Right main pulmonary+left subclavian artery thrombus & 1 & 0.4 \\
\hline & Right main pulmonary+left subsegmental artery thrombus & 1 & 0.4 \\
\hline & Total & 261 & 98.9 \\
\hline
\end{tabular}

\section{Table 3. Transthoracic echocardiography findings}

\begin{tabular}{|c|c|c|c|c|c|c|}
\hline \multicolumn{3}{|c|}{ Variables } & $\begin{array}{l}\text { Chest Diseases Clinic } \\
(\text { Mean } \pm \text { SD })\end{array}$ & ICU (Mean \pm SD) & Total $($ Mean \pm SD) & $\mathbf{p}$ \\
\hline \multicolumn{3}{|l|}{ PAP } & $42.50 \pm 13.73 \mathrm{mmHg}$ & $51.53 \pm 15.43 \mathrm{mmHg}$ & $45.90 \pm 15.01 \mathrm{mmHg}$ & $<0.001$ \\
\hline \multicolumn{6}{|c|}{ Mean \pm SD } & $\mathbf{p}$ \\
\hline \multirow{4}{*}{ PAP } & \multicolumn{2}{|c|}{ Thrombolytic therapy (rtPA) } & & $53.75 \pm 15.41 \mathrm{mmHg}$ & & \multirow{2}{*}{$<0.001$} \\
\hline & \multicolumn{2}{|c|}{ LMWH } & & $42.53 \pm 13.64 \mathrm{mmHg}$ & & \\
\hline & \multicolumn{3}{|c|}{ Half-dose thrombolytic therapy (rtPA) (50 mg) } & $50.63 \pm 13.52 \mathrm{mmHg}$ & & \multirow{2}{*}{0.156} \\
\hline & \multicolumn{3}{|c|}{ Full-dose thrombolytic therapy (rtPA) (100 mg) } & $56.98 \pm 17.95 \mathrm{mmHg}$ & & \\
\hline & & & Chest Diseases Clinic (n, \%) & $\operatorname{ICU}(\mathbf{n}, \%)$ & Total (n, \%) & $\mathbf{p}$ \\
\hline \multirow{2}{*}{\multicolumn{2}{|c|}{ RVD }} & No & $125(72.3)$ & $15(10.7)$ & $140(53.0)$ & \multirow{2}{*}{$<0.001$} \\
\hline & & Yes & $60(48.4)$ & $64(51.6)$ & $124(47.0)$ & \\
\hline \multirow{2}{*}{\multicolumn{2}{|c|}{$\begin{array}{l}\text { Paradoxical septal motion } \\
\text { with left-axis deviation }\end{array}$}} & No & $185(76.1)$ & $58(23.9)$ & $243(92.7)$ & \multirow{2}{*}{$<0.001$} \\
\hline & & Yes & $4(21.1)$ & $15(78.9)$ & $19(7.3)$ & \\
\hline & & & $\begin{array}{l}\text { Half-dose thrombolytic } \\
\text { therapy (rtPA) (50 mg) (n, \%) }\end{array}$ & $\begin{array}{l}\text { Full-dose thrombolytic therapy } \\
\text { (rtPA) }(100 \mathrm{mg})(\mathrm{n}, \%)\end{array}$ & Total (n, \%) & \\
\hline \multirow{2}{*}{\multicolumn{2}{|c|}{ RVD }} & No & $4(80.0)$ & $1(20.0)$ & $5(8.8)$ & \multirow{2}{*}{0.157} \\
\hline & & Yes & $21(40.4)$ & $31(59.6)$ & $57(91.2)$ & \\
\hline
\end{tabular}

RVD:Right ventricular dilatation, PAP:Pulmonary artery pressure, rtPA:Recombinant tissue plasminogen activator, LMWH:Low-molecular-weight heparin, SD: standard deviation

The patients comprised $206(78.0 \%)$ nonmassive, 17 (6.5\%) submassive, and 41(15.5\%) massive acute PTE (Table 1).Of the 264 patients, 57 (21.6\%) of them received $\mathrm{rtPA}$ and 207 (78.4\%) of them received LMWH therapy. In the rtPA group, 25 (43.9\%) of them received half-dose rtPA therapy $(50 \mathrm{mg})$ and $32(56.1 \%)$ of them received full-dose rtPA therapy $(100 \mathrm{mg})$. Treatmentrelated complications were observed in 14 (5.4\%) of 264 patients, including 7 (3.4\%) patients in the LMWH group and $7(13.0 \%)$ patients in the rtPA group.
Table 4 presents complete blood count parameters. Total mortality occurred in 8 (3.0\%) out of 264 patients. Hospital and ICU mortality were determined at $1.9 \%$, $1.1 \%$ respectively (Table $\mathbf{1}$ ). Risk factors for 90 -mortality included comorbidities, acquired risk factors, ICU hospitalization, increased APACHE II scores, presence of RVD on TTE, rtPA therapy, invasive and noninvasive mechanical ventilation, and persistent hypotension, tachycardia, and hypoxemia $(\mathrm{p}=0.008, \mathrm{p}=0.025, \mathrm{p}=0.001$ $, \mathrm{p}<0.001, \mathrm{p}=0.028, \mathrm{p}=0.035, \mathrm{p}<0.001, \mathrm{p}=0.001, \mathrm{p}<0.001$, 
$\mathrm{p}=0.028)$. Of these parameters, WBC, RDW, and MPV/ $\mathrm{RDW}$ ratio were found to be significant risk factors for 90 -day mortality $(\mathrm{p}=0.032, \mathrm{p}=0.041$, and $\mathrm{p}=0.039)$ (Table 5).

Risk factors for ICU requirement included acquired risk factors, dyspnea, dry cough, syncope, presence of RVD on TTE, paradoxical septal motion with left-axis deviation, PAP, rtPA therapy, invasive and noninvasive mechanical ventilation, use of vasopressors, and persistent hypotension and hypoxemia $(\mathrm{p}<0.001)$. Additionally, baseline $\mathrm{CBC}$ parameters including WBC, HB, HCT, $\mathrm{RDW}$, and NE were also found to be significant factors for ICU requirement (Table 6).

\begin{tabular}{|lcc|}
\hline \multicolumn{3}{|l|}{ Table 4. Complete blood count parameters } \\
\hline Variables & Mean \pm SD & Median $(\mathrm{DW})$ \\
\hline $\mathrm{WBC}\left(10^{3} / \mu \mathrm{L}\right)$ & $10.07 \pm 3.82$ & $21.00(25.00)$ \\
$\mathrm{HB}(\mathrm{g} / \mathrm{dL})$ & $13.27 \pm 1.91$ & $13.30(12.60)$ \\
$\mathrm{HCT}(\%)$ & $39.91 \pm 5.76$ & $40.00(38.30)$ \\
$\mathrm{PLT}(\mu \mathrm{L})$ & $269227.27 \pm 95667.25$ & $254000.00(672000.00)$ \\
$\mathrm{RDW}(\%)$ & $15.15 \pm 2.12$ & $14.60(13.80)$ \\
$\mathrm{NE}\left(10^{3} / \mu \mathrm{L}\right)$ & $7.30 \pm 3.65$ & $6.39(21.81)$ \\
$\mathrm{LY}\left(10^{3} / \mu \mathrm{L}\right)$ & $1.92 \pm 1.28$ & $1.71(12.60)$ \\
$\mathrm{MPV}(\mathrm{fL})$ & $8.31 \pm 1.25$ & $8.30(14.17)$ \\
$\mathrm{NE} / \mathrm{LY}$ ratio & $5.83 \pm 7.40$ & $3.57(77.21)$ \\
$\mathrm{PLT} / \mathrm{LY}$ ratio & $188234.83 \pm 149730.06$ & $155044.93(175000.00)$ \\
MPV/PLT ratio & $0.0000349 \pm 0.0000138$ & $0.0000327(0.0000724)$ \\
MPV/RDW ratio & $0.56 \pm 0.11$ & $0.55(1.03)$ \\
\hline WBC:White blood cell count, HB:Hemoglobin, HCT:Hematocrit, PLT:Platelet count, \\
RDW:Red blood cell distribution width, NE:Neutrophil count, LY:Lymphocyte count, \\
MPV:Mean platelet volume, SD: Standard deviation \\
\hline
\end{tabular}

Table 5. Risk factors for mortality

\begin{tabular}{|c|c|c|c|c|c|c|c|}
\hline \multirow{2}{*}{ Variables } & \multicolumn{3}{|c|}{ No mortality occurred } & \multicolumn{3}{|c|}{ Mortality occurred } & \multirow{2}{*}{$\mathbf{p}$} \\
\hline & n & Mean \pm SD & Median(DW) & $\mathbf{n}$ & Mean \pm SD & Median (DW) & \\
\hline Age & 256 & $62.58 \pm 1.59$ & $63.00(73.00)$ & 8 & $69.75 \pm 14.88$ & $72.00(44.00)$ & 0.212 \\
\hline APACHE II & 256 & $20.70 \pm 5.00$ & $20.00(24.00)$ & 8 & $28.71 \pm 3.64$ & $28.00(11.00)$ & $<0.001$ \\
\hline PAP & 256 & $46.67 \pm 15.15$ & $42.00(87.00)$ & 8 & $51.43 \pm 10.29$ & $50.00(30.00)$ & 0.322 \\
\hline $\begin{array}{l}\text { Duration of ICU stay } \\
\text { (days) }\end{array}$ & 256 & $3.71 \pm 3.27$ & $3.00(21.00)$ & 8 & $5.00 \pm 8.02$ & $2.00(22.00)$ & 0.404 \\
\hline $\begin{array}{l}\text { Duration of stay at } \\
\text { clinic (days) }\end{array}$ & 256 & $8.62 \pm 6.75$ & $7.00(88.00)$ & 8 & $4.13 \pm 7.36$ & $0.00(17.00)$ & 0.065 \\
\hline $\mathrm{WBC}\left(10^{3} / \mu \mathrm{L}\right)$ & 256 & $9.98 \pm 3.70$ & $9.30(23.35)$ & 8 & $12.93 \pm 6.28$ & $11.10(20.20)$ & 0.032 \\
\hline $\mathrm{HB}(\mathrm{g} / \mathrm{dL})$ & 256 & $13.29 \pm 1.90$ & $13.40(12.60)$ & 8 & $12.65 \pm 2.27$ & $11.80(6.90)$ & 0.354 \\
\hline HCT (\%) & 256 & $40.01 \pm 5.67$ & $40.10(38.30)$ & 8 & $36.69 \pm 7.89$ & $34.20(25.00)$ & 0.108 \\
\hline $\operatorname{PLT}(\mu \mathrm{L})$ & 256 & $270664.06 \pm 96458.89$ & $255000(672000)$ & 8 & $223250.00 \pm 49271.12$ & $218000.00(157000.00)$ & 0.168 \\
\hline RDW (\%) & 256 & $15.10 \pm 2.12$ & $14.60(13.80)$ & 8 & $16.66 \pm 1.93$ & $16.80(5.40)$ & 0.041 \\
\hline $\mathrm{NE}\left(10^{3} / \mu \mathrm{L}\right)$ & 256 & $7.22 \pm 3.55$ & $6.32(19.51)$ & 8 & $9.71 \pm 6.02$ & $7.53(18.90)$ & 0.058 \\
\hline $\operatorname{LY}\left(10^{3} / \mu \mathrm{L}\right)$ & 256 & $1.91 \pm 1.22$ & $1.74(12.60)$ & 8 & $2.23 \pm 2.54$ & $1.45(7.82)$ & 0.490 \\
\hline $\mathrm{fL}$ & 256 & $8.32 \pm 1.25$ & $8.30(14.17)$ & 8 & $7.90 \pm 1.14$ & $8.20(3.10)$ & 0.352 \\
\hline NE/LY ratio & 256 & $5.69 \pm 7.05$ & $3.56(77.21)$ & 8 & $10.37 \pm 14.95$ & $5.75(45.57)$ & 0.078 \\
\hline PLT/LY ratio & 256 & $188373.40 \pm 150855.91$ & $155044(175000.00)$ & 8 & $183800.83 \pm 115198.63$ & $169500.00(341484.15)$ & 0.932 \\
\hline MPV/PLT ratio & 256 & $0.00 \pm 0.00$ & $0.00(0.00)$ & 8 & $0.00 \pm 0.00$ & $0.00(0.00)$ & 0.741 \\
\hline MPV/RDW ratio & 256 & $0.56 \pm 0.11$ & $0.55(1.03)$ & 8 & $0.48 \pm 0.08$ & $0.47(0.25)$ & 0.039 \\
\hline
\end{tabular}

Table 6. Risk factors for ICU requirement

\begin{tabular}{|c|c|c|c|c|c|}
\hline \multirow{2}{*}{ Variables } & \multicolumn{2}{|c|}{ No ICU required } & \multicolumn{2}{|c|}{ ICU required } & \multirow{2}{*}{$\mathbf{p}$} \\
\hline & Mean \pm SD & Median (DW) & Mean \pm SD & Median (DW) & \\
\hline Age & $62.70 \pm 15.90$ & $62.00(71.00)$ & $63.04 \pm 16.21$ & $66.00(70.00)$ & 0.877 \\
\hline PAP & $42.24 \pm 13.53$ & $40.00(87.00)$ & $51.81 \pm 15.49$ & $50.00(60.00)$ & $<0.001$ \\
\hline $\mathrm{WBC}\left(10^{3} / \mu \mathrm{L}\right)$ & $9.53 \pm 3.52$ & $9.05(22.90)$ & $11.41 \pm 4.21$ & $11.25(23.05)$ & $<0.001$ \\
\hline $\mathrm{HB}(\mathrm{g} / \mathrm{dL})$ & $13.44 \pm 1.82$ & $13.50(12.60)$ & $12.84 \pm 2.07$ & $12.85(9.60)$ & 0.021 \\
\hline HCT (\%) & $40.37 \pm 5.44$ & $40.15(38.30)$ & $38.76 \pm 6.39$ & $38.90(32.00)$ & 0.039 \\
\hline $\operatorname{PLT}(\mu \mathrm{L})$ & $275723.40 \pm 97467.55$ & $257000.00(672000.00)$ & $253157.89 \pm 89665.31$ & $227500.00(437000.00)$ & 0.083 \\
\hline RDW (\%) & $14.97 \pm 2.14$ & $14.50(13.80)$ & $15.59 \pm 2.04$ & $15.05(9.90)$ & 0.032 \\
\hline $\mathrm{NE}\left(10^{3} / \mu \mathrm{L}\right)$ & $6.74 \pm 3.32$ & $6.10(19.40)$ & $8.68 \pm 4.07$ & $7.94(21.81)$ & $<0.001$ \\
\hline $\mathrm{LY}\left(10^{3} / \mu \mathrm{L}\right)$ & $1.92 \pm 1.27$ & $1.80(12.50)$ & $1.93 \pm 1.30$ & $1.55(8.13)$ & 0.925 \\
\hline MPV (fL) & $8.30 \pm 1.16$ & $8.30(10.45)$ & $8.33 \pm 1.45$ & $8.43(9.17)$ & 0.842 \\
\hline $\mathrm{NE} / \mathrm{LY}$ ratio & $5.28 \pm 5.85$ & $3.41(45.71)$ & $7.19 \pm 10.20$ & $4.50(76.66)$ & 0.059 \\
\hline PLT/LY ratio & $186749.15 \pm 119104.04$ & $154810.04(982500.00)$ & $191909.94 \pm 207911.52$ & $155061.63(1737310.92)$ & 0.8 \\
\hline MPV/PLT ratio & $0.00 \pm 0.00$ & $0.00(0.00)$ & $0.00 \pm 0.00$ & $0.00(0.00)$ & 0.132 \\
\hline MPV/RDW ratio & $0.56 \pm 0.11$ & $0.55(0.78)$ & $0.55 \pm 0.13$ & $0.55(0.72)$ & 0.268 \\
\hline
\end{tabular}

PAB:Pulmonary artery pressure, ICU:Intensive care unit, WBC:White blood cell count, HB:Hemoglobin, HCT:Hematocrit, PLT:Platelet count, RDW:Red blood cell distribution width, NE:Neutrophil count, LY:Lymphocyte count, MPV:Mean platelet volume, SD: Standard deviation 


\section{DISCUSSION}

Risk factors for 90-mortality included WBC, RDW, MPV/ RDW ratio, comorbidities, acquired risk factors, ICU hospitalization, increased APACHE II scores, presence of RVD on TTE, rtPA therapy, invasive and noninvasive mechanical ventilation, and persistent hypotension, tachycardia, and hypoxemia.

In Acute PTE, the mechanical obstruction of the pulmonary vascular bed caused by a thrombus localized to the pulmonary arteries leads to hemodynamic alterations, thereby leading to increased ventricular afterload and often resulting in RVD and hypokinesia. TTE is a bedside diagnostic tool commonly used in the detection of RVD and in making systemic thrombolytic treatment decisions (9). A previous study retrospectively evaluated the TTE findings of patients with massive PTE and detected RVD in $90.0 \%$, paradoxical septal motion with left-axis deviation in $65.0 \%$, and PAP $>40 \mathrm{mmHg}$ in $60.0 \%$ of the patients (10). Similarly, Serra et al. (11) retrospectively evaluated the TTE findings of PTE patients and revealed the baseline PAP as $39 \mathrm{mmHg}$. In our study, RVD was detected in $53.0 \%$ and paradoxical septal motion with left-axis deviation was detected in $7.3 \%$ of the patients. The TTE findings of the patients were retrospectively reviewed by an experienced cardiologist and patients with ventricular dysfunction and heart valve disease were excluded from the study. The mean PAP was $45.90 \pm 15.01 \mathrm{mmHg}$, which was higher than most of the values reported in the literature. This difference was attributed to the high prevalence of systemic thrombolytic therapy in our patients who had hemodynamic instability or in those who were hemodynamically stable but had RVD (21.5\%). Moreover, the mean PAP in our patients that were hospitalized in the Chest Diseases clinic was remarkably high $(42.50 \pm 13.73 \mathrm{mmHg})$, which could be associated with the fact that the TTE findings were examined by different cardiologists and by using different devices and that the TTE findings are not standardized and thus can be affected by hemodynamic alterations such as body weight and tachycardia. As most of the patients hospitalized in ICU were hemodynamically instable, their TTE findings were statistically significant compared to those of patients who were hospitalized in the Chest Diseases clinic, as consistent with the literature.

Intravenous thrombolytic agents can be used to reduce pulmonary perfusion and PAP and to restore right ventricular function in a prompt manner $(12,13)$. Current international guidelines propose that PTE patients with RVD and hemodynamic instability can be treated with systemic half-dose (rtPA $50 \mathrm{mg}$ ) or full-dose (rtPA 100 $\mathrm{mg}$ ) thrombolytic therapy based on the benefit/loss analysis for each patient $(14,15)$. In some studies, halfdose therapy has been shown to be as effective as full- dose therapy and has also been reported to promote angiographic revascularization and to provide an improvement in PAP similar to that of full-dose therapy. Streptokinase, urokinase, and rtPA are commonly used thrombolytic agents $(16,17)$. In our study, $57(21.5 \%)$ patients received rtPA as systemic thrombolytic therapy, among whom 25 (43.9\%) patients received half-dose rtPA $(50 \mathrm{mg})$ and $32(56.1 \%)$ patients received full-dose rtPA (100 mg). Acute PTE is available in studies that indicate the systemic thrombolytic therapy reguirement $4.2 \%$ and $18.0 \%(16,17)$. In our study, however, the prevalence of systemic thrombolytic therapy was higher than those reported in the literature, which could be associated with several factors including the high rate of ICU hospitalization (28.4\%), high APACHE II scores and PAP values, and high prevalence of vasopressor requirement, hemodynamic instability, and RVD in our patients. On the other hand, the prevalence of full-dose rtPA therapy was higher than that of half-dose rtPA therapy $(56.1 \%$ vs. $43.9 \%)(11,16,17)$. However, no significant difference was found between these two groups with regard to PAP and the prevalence of RVD, both of which are significant pathfinders in the decision-making processes regarding thrombolytic therapy and its dosage. This finding could be attributed to the prioritization of hemodynamic findings over TTE findings in the decision-making processes regarding thrombolytic therapy and its dosage.

PTE is a significant cause of mortality and morbidity. In previous studies, 90-day mortality rate has been reported as $58.3 \%$ in patients with massive PTE and as $15.1 \%$ in patients with submassive PTE $(11,18)$. In our study, massive (15.3\%) and submassive (6.5\%) PTE ratio has been lower than studies where 90 days of mortality have been evaluated.Hospital stay in patients with acute PTE has been reported to vary between 4 and 10 days $(19,20)$. In our study, 90-day mortality occurred in 8 (3.0\%) out of 264 patients. This rate was remarkably lower than those reported in the literature, which could be associated with the administration of systemic thrombolytic therapy in hemodynamically instable patients and the shorter duration of hospitalization among our ICU patients (3.83 \pm 3.87 days). In our study, mean duration of hospital stay was $8.49 \pm 6.80$ days and the mean duration of ICU stay was $3.83 \pm 3.87$ days. In our study, the heterogeneous patient groups and the high number of nonmassive acute PTE patients (nonmassive, massive and submassive) caused the number of hospital stay days to be high. No significant difference was found between these two values (hospital and ICU stay days) with regard to 90day mortality. In our study, it was thought that the data of patients without hemodynamic change may affect our results.Our results are compared to literature information in acute PTE, where 90 days mortality are evaluated. This finding could be associated with the low mortality 
rate in our patients. Additionally, the duration of hospitalization in our ICU patients was lower than those of many studies in the literature. On the other hand, the longer hospitalization period in the patients hospitalized in the chest diseases clinic compared to the patients hospitalized in ICU could be ascribed to the fact that most of the patients that were initiated on the LMWH therapy subsequently used oral anticoagulants (warfarin) as maintenance therapy and the patients were discharged after ensuring patient compliance with the anticoagulant warfarin therapy. Risk factors for 90-mortality included comorbidities, acquired risk factors, ICU hospitalization, increased APACHE II scores, presence of RVD on TTE, rtPA therapy, invasive and noninvasive mechanical ventilation, and persistent hypotension, tachycardia, and hypoxemia.In contrast, risk factors for ICU requirement included acquired risk factors, dyspnea, dry cough, syncope, presence of RVD on TTE, paradoxical septal motion with left-axis deviation, PAP, rtPA therapy, invasive and noninvasive mechanical ventilation, use of vasopressors, and persistent hypotension and hypoxemia. Taken together, these findings implicate that hemodynamic parameters were risk factors for both mortality and ICU requirement, as consistent with the literature.

In the pathophysiology of PTE, inflammatory mediators have been shown to be upregulated and to interact with coagulation factors. There is no cellular infiltration in the pulmonary artery during the acute stage while leukocyte infiltration occurs within the subsequent three hours and two days, which decreases on the fourth day and returns to baseline levels on the eighth day (18). Inflammatory markers including WBC, HB, NE, LY, PLT, RDW, and MPV, which are inexpensive and standard biomarkers that are routinely measured in $\mathrm{CBC}$, have recently become a major research area in the evaluation of PTE $(21,22)$. In those studies, serum levels of these markers have been shown to decrease or increase in PTE. Additionally, increased NE counts have been associated with subclinical inflammation and decreased LY counts have been attributed to the elevated glucocorticoid levels induced by sympathetic activation (22). They are used either in isolation or in ratios such as NE/LY and PLT/LY ratios for predicting mortality and risk factors in acute PTE. A previous review suggested that the NE/ LY ratio could be used in predicting mortality in PTE patients although there are some studies presenting controversial results regarding the use of NE/LY and PLT/LY ratios in such predictions (23). PLT plays a central role in thromboembolic diseases by secreting prothrombotic and proinflammatory molecules. MPV is a biomarker which represents average platelet size and has been shown to indicate macrothrombocytes when elevated and to play a role in venous thrombosis
(24). RDW reflects the degree of heterogeneity of RBC size and has been shown to be elevated in conditions leading to increased levels of inflammatory markers such as CRP. Additionally, an elevated RDW indicates immature $\mathrm{RBC}$ production in the bone marrow and is known to be associated with the changes in blood viscosity and acute inflammation $(25,26)$. In a previous case-control study, Payman et al. (27) evaluated 173 PTE patients and reported that the PLT counts were not elevated in any patient. In contrast, another study evaluated patients with acute DVT and reported that the MPV/PLT ratio and the MPV values were higher in the patients compared to control subjects (28). Kovacs et al. (7) reviewed multiple studies that evaluated DVT patients and revealed that the MPV values were significantly elevated in the patients compared to control subjects and this elevation was found to be statistically significant in patients with acute PTE in subgroup analyses. Some other studies suggested that MPV is an independent risk factor in predicting early mortality in acute PTE $(28,29)$. Similarly, a prospective study evaluated 378 patients with acute PTE and reported that baseline RDW value was an independent risk factor for predicting 17-month mortality (30). Some previous studies proposed low MPV and RDW values for predicting thrombus risk and mortality while some others proposed high values, serial measurements, and different cutoff values for different groups of the same disease In our study, baseline serum parameters were measured in CBC and the NE/LY, PLT/LY, MPV/ PLT, and MPV/RDW ratios were calculated based on these parameters (31). We used these parameters and ratios to investigate their role in predicting 90-day mortality and ICU requirement. The measurements indicated that although the NE and WBC counts were higher than the reference values, no significant decrease was found in the LY counts, unlike in other studies (22). Moreover, WBC, NE, HB, HCT, and RDW were found to be significant risk factors for predicting ICU requirement and $\mathrm{WBC}, \mathrm{RDW}$, and the $\mathrm{MPV} / \mathrm{RDW}$ ratio were significant risk factors for predicting 90day mortality. Accordingly, RDW was found to be a significant risk factor for predicting both mortality and ICU requirement, which could be attributed to the high prevalence of hemodynamic instability, blood viscosity changes, and acute inflammation in our patients. Additionally, the PLT, NE/LY, PLT/LY, and MPV/PLT ratios were insignificant risk factors and the PLT and LY counts were in normal ranges, which could be ascribed to numerous factors including the retrospective nature of the study, nonstandardization of the methods used in collecting baseline serum samples at hospital presentation (i.e. the absence of a fixed timing schedule for the collection of serum samples), failure to analyze 
time-dependent biomarkers such as MPV within one hour after blood collection, and the absence of serial measurements. Accordingly, we consider that serial measurement of inflammatory markers at presentation (baseline) and at 3,24, and 48 hours after presentation could have revealed an increase in these parameters and their ratios. This assumption is relies on the literature data that indicated that acute inflammation begins to increase three hours after the onset of the symptoms and remains elevated for up to $48 \mathrm{~h}$ (18). Accordingly, in our study, the mean $\mathrm{WBC}$ and $\mathrm{NE}$ values showed an early increase while the MPV, PLT, and LY values showed a normal increase, which could be explained by the fact that MPV, PLT, and LY typically begin to increase at a later period compared to $\mathrm{WBC}$ and NE. Based on these findings, we suggest that the levels of these parameters in acute PTE should be standardized by serial measurements. We also consider that RDW can be an early marker of ICU requirement and mortality in acute PTE and further studies involving risk analysis are needed to investigate the role of RDW in acute PTE.

\section{CONCLUSION}

The results indicated that TTE findings, baseline hemodynamic parameters and symptoms, rtPA therapy, and $\mathrm{CBC}$ parameters including $\mathrm{WBC}, \mathrm{NE}$, and $\mathrm{RDW}$ are significant risk factors for predicting both mortality and ICU requirement. Further studies are needed to investigate the role of RDW and the MPV/RDW ratio in the risk analysis of PTE and in patients with thrombus or those with a risk of thrombus development.

\section{ETHICAL DECLARATIONS}

Ethics Committee Approval: Ethical approval was obtained from the Pamukkale University Noninterventional Clinical Research Ethics Committee (Date: 21.05.2019, Decision No: 10). Health Sciences University, Atatürk Chest Diseases and Chest Surgery Education and Research Hospital, Specialization in Medicine Education Board approval (Date: 27.02.2020, Decision No: 664).

Informed Consent: Because the study was designed retrospectively, no written informed consent form was obtained from patients.

Referee Evaluation Process: Externally peer-reviewed.

Conflict of Interest Statement: The authors have no conflicts of interest to declare.

Financial Disclosure: The authors declared that this study has received no financial support.

Author Contributions: All of the authors declare that they have all participated in the design, execution, and analysis of the paper, and that they have approved the final version.

\section{REFERENCES}

1. Konstantinides SV, Meyer G, Becattini C, et al. 2019 ESC Guidelines for the diagnosis and management of acute pulmonary embolism developed in collaboration with the European Respiratory Society (ERS): The Task Force for the diagnosis and management of acute pulmonary embolism of the European Society of Cardiology (ESC). Eur Respir J 2019: 54.

2. Kusumoto FM, Schoenfeld MH, Barrett C, et al. 2018 ACC/AHA/ HRS Guideline on the Evaluation and Management of Patients With Bradycardia and Cardiac Conduction Delay: Executive Summary: A Report of the American College of Cardiology/ American Heart Association Task Force on Clinical Practice Guidelines, and the Heart Rhythm Society. J Am Coll Cardiol 2019; 74: 932-87.

3. Weitz JI, Chan NC. Novel antithrombotic strategies for treatment of venous thromboembolism. Blood 2020; 135: 351-9.

4. Zhou XY, Chen HL, Ni SS. Red cell distribution width in predicting 30-day mortality in patients with pulmonary embolism. J Crit Care 2017; 37: 197-201.

5. Agarval S. Red cell distribution width, inflammatory markers and cardiorespiratory fitness: Results from the National Health and Nutrition Examination Survey. Indian Heart J 2012; 64: 380-7.

6. Montagana M, Cervelin G, Meschi T, Lippi G. The role of red blood cell distribution width in cardiovascular and thrombotic disorders. Clin Chem Lab Med 2011; 50: 635-41.

7. Kovacs S, Csiki Z, Zsori KS, Shemirani AH. Characteristics of platelet count and size and diagnostic accuracy of mean platelet volUme in patients with venous thromboembolism. A systematic review and meta-analysis. Platelets 2019; 30: 139-47.

8. Falk E, Shah PK, Fuster V.Coronary plague disruption.Circulation 1995; 92: 657-71.

9. Ishaaya E, Tapson VF. Advances in the diagnosis of acute pulmonary embolism. F1000Res 2020; 9.

10. Lee K, Kwon O, Lee EJ, et al. Prognostic value of echocardiographic parameters for right ventricular function in patients with acute nonmassive pulmonary embolism. Heart Vessels 2019; 34: 1187-95.

11.Serra W, Crisafulli E, Syerzellati N, et al. Transthoracic echocardiography and chest computed tomography arteriography in patients with acute pulmonary embolism: a two-year followup study. Respiration 2016; 92: 235-40.

12. Hepburn-Brown M, Darvall J, Hammerschlag G. Acute pulmonary embolism: a concise review of diagnosis and management. Intern Med J 2019; 49: 15-27.

13. Manuel A, Aufico A, Africanı R, et al. Clinical profile, management and outcomes of patients with pulmonary embolism: a retrospective tertiary centre study in Angola. Cardiovasc J A fr 2017; 28 356-61.

14. Keller K, Hobohm L, Ebner M, et al. Trends in thrombolytic treatment and outcomes of acute pulmonary embolism in Germany. Eur Heart J 2020 ; 41: 522-29.

15. Quezada A, Jimenez D, Bikdeli B, et al. Systolic blood pressure and mortality in acute symptomatic pulmonary embolism. Int J Cardiol 2020; 302: 157-63.

16. Di Nisio M, van Es N, Büller HR. Deep vein thrombosis and pulmonary embolism. Lancet 2016; 388: 3060-73.

17. Verma B, Singh AK. Use of reteplase for thrombolysis in patients with massive pulmonary embolism diagnosed by bedside transthoracic echocardiography: A retrospective study of safety and efficacy. J Family Med Prim Care 2019; 8: 3155-9.

18. Goldhaber SZ, Buring JE, Lipnick RJ, Hennekens CH. Pooled analyses of randomized trials of streptokinase and heparin in phlebographically documented acute deep venous thrombosis. Am J Med 1984; 76: 393-7.

19. Fanikos J, Rao A, Seger AC, et al. Hospital Costs of Acute Pulmonary Embolism. Am J Med 2013; 126: 127-32. 
20.Donadini MP, Dentali F, Castellaneta M, et al. Pulmonary embolism prognostic factors and length of hospital stay: A cohort study. Thromb Res 2017; 156: 155-9.

21. Omar HR, Mirsaeidi M, Rashad R, et al. Association of serum albumin and severity of pulmonary embolism. Medicana (Kaunas) 2020; 9: 56.

22. Bazouki G, Letsas KP, Vlachos K, et al. Simple hematological predictors of $\mathrm{AF}$ recurrence in patients undergoing atrial fibrillation ablation. J Geriatr Cardiol 2019; 16: 671-5.

23. Galliazzo S, Nigro O, Bertü L, et al. Prognostic role of neutrophils to lymphocytes ratio in patients with acute pulmonary embolism: a systematic review and metaanalysis of the literatüre. Intern Emerg Med 2018; 13: 603-8.

24. Farah R, Nseir W, Kagansky D, Khamisy-Farah R. The role of neutrophil-lymphocyte ratio and mean platelet volüme in detecting patients with acute venous thromboembolism. J Clin Lab Anal 2020; 34: e23010.

25. Hammons L, Filopel J, Steiger D, Bondarsky E. A narrative review of red blood cell distribution width as a marker for pulmonary embolism. J Thromb Thrombolysis 2019; 48: 638-47.

26. Yu FT, Armstrong JK, Tripette J, Meiselman HJ, Cloutier G. A local increase in red blood cell aggreagation ca trigger deep vein thrombosis: evidence based on quantitative cellular ultrasound imaging. J Thromb Haemost 2011; 9: 481-8.

27. Moharamzadeh P, Rahmani F, Foroughifar S, Shahsavarinia K. Reliability of Platelet Indices for Diagnosing Pulmonary Embolism; a Brief Report. Adv J Emerg Med 2019; 3: e27.

28. Han JS, Park TS, Cho SY, Joh JH, Ahn HJ. Increased mean platelet volume and mean platelet volüme/platelet count ratio in Korean patients with deep vein thrombosis. Platelets 2013; 24: 590-3.

29. Kostrubiec M, Labyk A, Pedowska-Wloszek J, et al. Mean platelet volume predicts early death in acute pulmonary embolism. Heart 2010; 96: 460-5.

30. Kheirkman-Saberghadam S, Jenab Y, Ghoreyski-Hefzabad, et al. Association between elevated red blood cell distribution width and long-term mortality in acute pulmonary embolism.Turk J Med Sci 2018; 48: 318-23.

31.Han YQ, Yan L, Zhang L, et al. Red blood cell distribution width provides additional prognostic value beyond severity scores in adult critical illness. Clin Chim Acta 2019; 498: 62-6. 